\title{
Evaluation of Iris Recognition System on Multiple Feature Extraction Algorithms and its Combinations
}

\author{
Ashwini M B \\ Department of \\ Computer Science, \\ MMK \& SDM Mahila \\ Maha Vidyalaya, K M \\ Puram, Mysore, \\ Karnataka, India
}

\author{
Mohammad Imran \\ Department of CCSIT, \\ King Faisal University \\ Al Ahsa, KSA
}

\author{
Fawaz Alsaade \\ Department of CCSIT, \\ King Faisal University \\ Al Ahsa, KSA
}

\begin{abstract}
Multi-algorithmic approach to enhancing the accuracy of iris recognition system is proposed and investigated. In this system, features are extracted from the iris using various feature extraction algorithms, namely LPQ, LBP, Gabor Filter, Haar, Db8 and Db16. Based on the experimental results, it is demonstrated that Mutli-algorithms Iris Recognition System is performing better than the unimodal system. The accuracy improvement offered by the proposed approach also showed that using more than two feature extraction algorithms in extracting the iris system might decrease the system performance. This is due to redundant features. The paper presents a detailed description of the experiments and provides an analysis of the performance of the proposed method.
\end{abstract}

Keywods: Biometrics, Iris, Unimodal, Multiple Algorithms, Feature Level Fusion, Performance of Algorithms

\section{INTRODUCTION}

Biometrics is the science of recognizing a person on the basis of physical or behavioral characteristics. Psychological characteristics are characteristics that are gathered or learned during time (e. g. signature, gait, typing dynamics, voice features etc.). Physical characteristics are characteristics that are genetically implied and possibly influenced by the environment (e. g. face, iris, retina, finger, vascular structure etc.). Iris biometric is employed in this work because of its distinctive and uniqueness in each person including identical twins and each eye. There are number of properties that enhance the suitability of the iris for use in automatic identification. One important feature is that the iris is inherently isolated and protected from the external environment. Moreover, being an internal organ of the eye, behind the cornea and the aqueous humor; it is almost impossible to surgically modify the iris without unacceptable risk of damage. Also, its physiological response to light provides a natural test against fake irises [11].

Iris provides many interlacing minute characteristics such as freckles, coronas, stripes, furrows, crypts and so on. It is essentially stable over a person's life. Iris-based personal identification systems are noninvasive to their users. The technology of Iris recognition has some advantages and disadvantages which make it appropriate to use in some application and not 
to other applications[12]. Some of the advantages of iris biometrics are as follows: a) Iris is very accurate biometric. It has low false acceptance rate, which is important in security aspect. Therefore iris might be a good biometric for identification applications. b) The sensing of the biometric is without physical contact and it is convenient for the users because the iris pattern acquisition process uses unnoticeable and distant cameras. c)Iris recognition system has low cost training. d) Iris technology has received little negative press and so is more generally accepted biometric identifier[2].

There are various unimodal iris recognition systems exists in real world, unimodal system means rely on single source of information for verification/identification. However, Multibiometric system relies on the evidence presented by multiple sources of biometric information. Based on the nature of these sources, a Multibiometric system can be classified into one of the following six categories: multi-sensor, multi-algorithm, multi-instance, multi-sample, multimodal and hybrid systems [ ]. In some cases, invoking multiple feature extraction and/or matching algorithms on the same biometric data can result in improved matching performance. Multi-algorithm system consolidates the output of multiple feature extraction algorithms, or that of multiple matchers operating on the same feature set. These systems do not necessitate the deployment of new sensors and, hence, are cost-effective compared to other types of multibiometric systems. On the other hand, the introduction of new feature extraction and matching modules can increase the computational complexity of these systems [3].

The work presented in this paper aims to enhancing the security of iris recognition system using multi-algorithmic approach. This is achieved by fusing the data acquired at the feature level and applying the K-Nearest Neighbor classifier(K-NN). The rest of the paper is structured as follows. Section 2 presents related works. Section 3 introduces the proposed approach for multi-algorithm iris recognition system. Section 4 describes the experimental investigations, and the overall conclusions are presented in Section 5.

\section{RELATED WORKS}

Karen et al [1] states that, genetically identical irises have texture similarity that is not detected by iris biometrics. By performing experimental studies on left and right irises of the same person, it results that are as different as irises of unrelated people. Similarly, in terms of iris biometric matching, the eyes of identical twins are as different as irises of unrelated people. The experiments done based on texture feature extraction methods, this highlight the difference between automated biometric technology and human viewers. This work suggests that human examination of pairs of iris images for forensic purposes may be feasible and results of experiments suggest that development of different approaches to automated iris image analysis could be used to improve current iris biometric technology. Vijaya et al [4] states that generally algorithm is divided into four steps, such as. localization, Normalization, Feature Extraction and Matching. Doughman's approach is there with highest accuracy of $99.9 \%$ and Kaushik Rai's approach is also promising in state of the art, only thing that can be done is to decrease the computational time and number of features to obtain the same efficiency.

Libor et al [5] presents an automatic segmentation algorithm that remove iris region from an eye image and isolate eyelid, eyelash and reflection areas. The iris region was normalized into a rectangular block with constant dimensions to account for imaging inconsistencies. However, the phase data from 1D Log-Gabor filters was extracted and quantized to four levels to encode the unique pattern of the iris into a bit-wise biometric template. The Hamming distance was employed for classification of iris templates, and two templates were found to match if a test of statistical independence was failed. The system performed with perfect recognition on a set of 75 eye images; however, tests on another set of 624 images resulted in false accept and false reject rates of $0.005 \%$ and $0.238 \%$ respectively. Therefore, iris recognition is shown to be a 
reliable and accurate biometric technology. Somnath et al [6] present a novel and efficient approach to extract iris features and matching technique to compare iris features. This approach uses Daubechies wavelet transform with four coefficients. Daubechies wavelet transform is easy to compute and fast compared to the other methods on texture analysis. Further, Daubechies wavelet transform allows to keep the count of feature vectors into a significantly lesser numbers.

Fenghua et al [7] presents a multi-algorithmic fusion approach for iris recognition, which combines phase based algorithm and zerocrossing based algorithm. The two algorithms are fused at the matching score level using SVM fusion strategy. The experimental results on CASIA and UBIRIS iris database show that the proposed approach can improve the recognition performance compared with the individual recognition algorithm and SVM based fusion strategy can give the better performance than the traditional fusion strategies.

\section{METHODS AND MATERIALS}

In this section, we emphasis on different iris recognition methods which are explored in state of the art approaches. Brief details of different iris biometric technology methods as well as feature extraction algorithms are explored here.

\subsection{Iris Recognition Methods}

a. Phase-based method: It recognizes iris patterns based on phase information which is independent of image contrast and illumination. The first complete, commercially available phase-based iris recognition system was designed and patented in 1994 by J.Daugman [15].

b. Texture-analysis based method: Wildes proposed iris recognition based on texture analysis. High quality iris images were captured using silicon intensified target camera coupled with a standard frame grabber and resolution of $512 \times 480$ pixels[12].

c. Zero-Crossing representation method: This method was developed by Boles. It represents features of the iris at different resolution levels based on the wavelet transform zero-crossing. The algorithm is translation, rotation and scale invariant. The input images are processed to obtain a set of 1D signals and its zero crossing representation based on its dyadic wavelet transform[9].

d. Intensity variations: An Iris recognition system developed by $\mathrm{Li} \mathrm{Ma}$ and characterized by local intensity variations. The sharp variation points of iris patterns are recorded as features[9].

e. Independent Component Analysis: The iris recognition system developed by Ya-Ping Huang adopts Independent Component Analysis (ICA) to extract iris texture features[11].

f. Iris authentication based on Continuous Dynamic Programming: The technique proposed by Radhika authenticates iris based on kinematic characteristics, acceleration. Pupil extraction begins by identifying the highest peak from the histogram which provides the threshold for lower intensity values of the eye image [12].

\subsection{Feature extraction algorithms}

In this paper, five different feature extraction algorithms have been used to extract the features prior to feature level fusion for the iris biometric. These are:

\section{a) Local Binary Pattern (LBP)}

Local Binary Pattern is an efficient method used for feature extraction and texture classification. LBP was first introduced by Ojala[8]. The LBP operator was introduced as a complementary measure for local image contrast, and it was developed as a grayscale invariant pattern measure adding complementary information to the "amount" of texture in images. LBP is ideally suited for applications requiring fast feature extraction and texture classification[13]. Due to its discriminative power and computational simplicity, the LBP texture operator has become a popular approach in various applications, including visual inspection, image retrieval, remote sensing, 
biomedical image analysis, motion analysis, environmental modeling, and outdoor scene analysis[3] .

\section{b) Local Quantization(LPQ)}

Ojansivu et al. [8] proposed a new descriptor for texture classification named as Local phase quantization (LPQ) that is robust to image blurring. The descriptor utilizes phase information computed locally in a window for every image position. The phases of the four lowfrequency coefficients are computed. These coefficients are decorrelated and uniformly quantized in an eight-dimensional space. The LPQ is in frequency domain, which contained partial contrast information of the image. However that is not enough to describe the texture features without contrast information in spatial domain. In LPQ algorithm, it firstly transformed the image from spatial domain to frequency domain to find the phase information coefficient for every single point from the image. Then the covariance between the adjacent pixels is computed.

\section{c) Haar Wavelet}

Haar functions have been used from 1910 when they were introduced by the Hungarian mathematician Alfred Haar[9]. Haar wavelet is discontinuous, and resembles a step function. It represents the same wavelets Daubechiesdb1. In mathematics, the Haar wavelet is a certain sequence of functions. Haar used these functions to give an example of a countable orthonormal system for the space of squareintegrable functions on the real line. The study of wavelets, and even the term "wavelet", did not come until much later. The technical disadvantage lies in the discontinuity of Haar wavelet, and therefore it is not differentiable. This property can, however, be an advantage for the analysis of signals with sudden transitions, such as monitoring of tool failure in machines.

\section{d) Gabor Filter}

In image processing, a Gabor filter, named after Dennis Gabor, is a linear filter used for edge detection[10]. Frequency and orientation representations of Gabor filters are similar to those of the human visual system, and they have been found to be particularly appropriate for texture representation and discrimination. In the spatial domain, a 2D Gabor filter is a Gaussian kernel function modulated by a sinusoidal plane wave. The Gabor filters are self-similar: all filters can be generated from one mother wavelet by dilation and rotation.J. G. Daugman discovered that simple cells in the visual cortex of mammalian brains can be modeled by Gabor functions. Thus, image analysis by the Gabor functions is similar to perception in the human visual system.

\section{e) Daubechies(db) wavelet}

Daubechies constructed the first wavelet family of scale functions that are orthogonal and have finite vanishing moments, i.e., compact support [9]. This property insures that the number of non-zero coefficients in the associated filter is finite. This is very useful for local analysis. The Haar wavelet is the basis of the simplest wavelet transform. It is also the only symmetric wavelet in the Daubechies family and the only one that has an explicit expression in discrete form. Haar wavelets are related to a mathematical operation called Haar transform, which serves as a prototype for all other wavelet transforms.

For the Daubechies wavelet transforms, the scaling signals and wavelets have slightly longer supports, i.e., they produce averages and differences using just a few more values from the signal. This slight change, however, provides a tremendous improvement in the capabilities of these new transforms. They provide us with a set of powerful tools for performing basic signal processing tasks. This family of wavelets with one parameter, due to Daubechies is the first one to make it possible to handle orthogonal wavelets with compact support and arbitrary regularity.

\section{RESULTS AND DISCUSSION}

This section, deals with the investigation consequences of combining different biometric feature extraction algorithm. Specifically at feature level fusion, Min-Max normalization rule 
to measure the performance of multi-algorithms system. In all the experiments, performance is measured in terms of Recognition rate, Recall,

\begin{tabular}{|c|c|c|c|c|c|}
\hline $\begin{array}{c}\text { Feature } \\
\text { extraction }\end{array}$ & LPQ & Gabor & Haar & Db8 & LBP \\
\hline $\begin{array}{c}\text { Recognition } \\
\text { rate }\end{array}$ & 89 & 68 & 66.5 & 73 & 61 \\
\hline Recall & 89 & 68 & 66.5 & 73 & 61 \\
\hline Precision & 90.66 & 66.91 & 68.32 & 75.08 & 63.23 \\
\hline F-measure & 89.82 & 67.45 & 67.39 & 74.02 & 62.09 \\
\hline
\end{tabular}

Precision and F-measure. First the performance of a single modality biometric system is measured; later the results for multi-algorithmic biometric system are evaluated.

CASIA Iris Image Database Version 1.0 (CASIA-IrisV1) is used for experimentations, it includes 756 iris images from 108 eyes[14]. For each eye, 7 images are captured in two sessions with our self-developed device CASIA close-up iris camera, where three samples are collected in the first session and four in the second session.

According to our experimental study, In table:1, it is found that as individual algorithm LPQ, Gabor Filter, Haar, Db8 and LBP independently results in $89 \%, 68 \%, 66.5 \%, 73 \%$ and $61 \%$ accuracy respectively. Performance of LPQ is the superior compare to other feature extraction methods.

By analyzing the results in Table-2, it can be observed that; The fusion of two feature extraction algorithms at feature level gives higher accuracy than single feature extraction method. We can also infer that, combination of LPB and LPQ algorithms outperforms the other existing combinations set.

However, Db8 and Haar algorithms combination is the least performance even compare to a single algorithm of LPQ. From Table -3, we can observe that there is no such impact in performance. Compare to its previous two algorithm fusion, however one can find the reduction in accuracy by introducing third feature extraction algorithm. Hence, choosing best combination plays a major role than increase the feature extraction algorithms.

Table-1: Performance of Single feature extraction algorithm

Table-2: Performance of two feature extraction algorithm

\begin{tabular}{|c|c|c|c|c|c|}
\hline $\begin{array}{c}\text { Algorithm } \\
\mathbf{1}\end{array}$ & $\begin{array}{c}\text { Algorithm } \\
\mathbf{2}\end{array}$ & Recognition & Recall & Precision & $\begin{array}{c}\text { F- } \\
\text { measure }\end{array}$ \\
\hline Db8 & Haar & 85.5 & 85.5 & 87.7 & 86.6 \\
\hline Db8 & LPQ & 92.0 & 92.0 & 90.6 & 91.3 \\
\hline Db8 & LBP & 91.5 & 91.5 & 91.1 & 91.3 \\
\hline Db8 & Gabor & 84.5 & 84.5 & 84.8 & 84.6 \\
\hline Haar & LPQ & 92.0 & 92.0 & 90.5 & 91.2 \\
\hline Haar & Gabor & 85.0 & 85.0 & 84.7 & 84.8 \\
\hline Haar & LBP & 90.5 & 90.5 & 90.2 & 90.3 \\
\hline LPQ & LBP & 95.0 & 95.0 & 93.8 & 94.4 \\
\hline LPQ & Gabor & 89.0 & 89.0 & 89.1 & 89.0 \\
\hline & LBP & 88.0 & 88.0 & 86.5 & 87.2 \\
\hline & & & & & \\
\hline & & & & & \\
\hline
\end{tabular}

Table-3: Performance of three feature extraction algorithm

\begin{tabular}{|c|c|c|c|c|c|c|}
\hline $\begin{array}{c}\text { Algorithm } \\
\mathbf{1}\end{array}$ & $\begin{array}{c}\text { Algorithm } \\
\mathbf{2}\end{array}$ & $\begin{array}{c}\text { Algorithm } \\
\mathbf{3}\end{array}$ & Recognition & Recall & Precision & $\begin{array}{c}\text { F- } \\
\text { measure }\end{array}$ \\
\hline LBP & LPQ & Db8 & 93.5 & 93.5 & 92 & 92.8 \\
\hline Db8 & Haar & LPQ & 90.5 & 90.5 & 89.8 & 90.14 \\
\hline Db8 & Haar & LBP & 90 & 90 & 90.4 & 90.2 \\
\hline Haar & Gabor & LPQ & 90.5 & 90.5 & 89.8 & 90.1 \\
\hline Haar & Gabor & LBP & 88.5 & 88.5 & 88.2 & 88.3 \\
\hline LPQ & Gabor & LBP & 94.5 & 94.5 & 93.7 & 94.1 \\
\hline
\end{tabular}


From the analysis of experimental results and observations, it can be concluded that; The performance of fusion of three feature extraction algorithms, at feature level on feature normalization of Min-Max rule. Does not have expected performance improvement over the two feature extraction algorithms. Fusion of three algorithms in iris recognition, the performance is same or decreased when compared to the performance of fusion of two algorithms. One such case in our experiment is fusion of LBP and LPQ has 95\% accuracy, when we introduce one more feature extraction algorithm to this combination, the performance is decreased drastically. The only exception is when fusing three algorithms of combination Db8 Haar and LPQ, which gained some good performance when compared to fusion of two algorithms. The performance of fusion of three algorithms, does not have performance improvement over the fusion of two which are the same performance in the best case. This is due to fusion of multiple redundant features at feature level, which also causes performance degradation of biometric system.

\section{REFERENCES}

[1] Karen Hollingsworth , Kevin W. Bowyer, Stephen Lagree, Samuel P. Fenker, Patrick J. Flynn "Genetically identical irises have texture similarity that is not detected by iris biometrics" Computer Science and Engineering Department, University of Notre Dame, Notre Dame, IN 46556, United States.

[ 2] R. Wheeler, S. Aitken, "Multiple algorithms for fraud detection", Artificial Intelligence Applications Institute, The University of Edinburgh, 80 South Bridge, Edinburgh EH1 1HN, Scotland, UK.

[3] Chowhan.S.S and G.N.Shinde, "Iris Biometrics Recognition Application in Security Management", *COCSIT, Ambajoagai Road, Latur413512, (M.S.) India,Indira Gandhi College, CIDCO, Nanded431602,(M.S.) India.

[4] S V Sheela, P A Vijaya, "Iris Recognition Methods - Survey", International Journal of
Computer Applications (0975 - 8887) Volume 3 - No.5, June 2010.

[5] Libor Masek, "Recognition of Human Iris Patterns for Biometric Identification", The University of Western Australia, 2003.

[6] SomnathDey and DebasisSamanta, "Improved Feature Processing for Iris Biometric Authentication System", International Journal of Electrical and Electronics Engineering 4:2 2010

[7] Fenghua Wang, Jiuqiang Han, Xianghua Yao, "iris recognition based on multialgorithmic fusion", WSEAS TRANSACTIONS ON INFORMATION SCIENCE \& APPLICATIONS manuscript received may 20, 2007; revised august 10 , 2007.

[ 8] Ojansivu, V., Heikkilä, J.: Blur Insensitive Texture Classification Using Local Phase Quantization. In Elmoataz, A., Lezoray, O., Nouboud, F., Mammass, D. (eds.) ICISP 2008 2008, LNCS, vol. 5099, pp. 236-243. Springer, Heidelberg (2008).

[9] Ayra Panganiban, Noel Linsangan and FelicitoCaluyo, "Wavelet-based Feature Extraction Algorithm for an Iris Recognition System", Journal of Information Processing Systems, Vol.7, No.3, September 2011.

[10]C. Sanchez-Avila, R. Sanchez-Reillo, "Two different approaches for iris recognition using Gabor filters and multiscale zerocrossing representation", Pattern Recognition Society, July 2004 (231 - 240).

[11]S V Sheela, P A Vijaya, "Iris Recognition Methods - Survey", International Journal of Computer Applications (0975 - 8887) Volume 3 - No.5, June 2010.

[12]Hugo Proenca and Luis A. Alexandre, "Iris Recognition: An Analysis of the Aliasing Problem in the Iris Normalization Stage", IEEE Proceedings of the 2006 International Conference on Computational Intelligence and Security - CIS 2006, Guangzhou, China, November 3-6, 2006, vol. 2, page. 1771-1774.

[13]Ahonen, T.; Hadid, A.; Pietikainen, M., "Face Description with Local Binary Patterns: Application to Face Recognition," Pattern Analysis and Machine Intelligence, 


\section{International Journal of Computer Applications Technology and Research}

Volume 4- Issue 8, 592 - 598, 2015, ISSN: 2319-8656

IEEE Transactions on , vol.28, no.12, pp.2037,2041, Dec. 2006

[14]Casia iris database. http://http://www.cbsr.ia.ac.cn/IrisDatabase. $\underline{\mathrm{html}}$
[ 15]John Daugman:Demodulation by ComplexValued Wavelets for Stochastic Pattern Recognition. IJWMIP 1(1): 1-17 (2003) 